\title{
BANCOS DE Sargassum cymosum (PHAEOPHYCEAE) NA ENSEADA DE ARMAÇÃO DO ITAPOCOROY, PENHA, SC: BIOMASSA E RENDIMENTO EM ALGINATO
}

\author{
MAFRA Jr., L.L. \& S.R. CUNHA \\ Laboratório de Ecologia da Vegetação Costeira - Centro de Ciências Tecnológicas da \\ Terra e do Mar - Universidade do Vale do Itajaí (CTTMar/UNIVALI), Itajaí, SC. \\ e-mail: mafrajr@cttmar.univali.br; simone@cttmar.univali.br
}

\section{RESUMO}

\begin{abstract}
A macroalga Sargassum forma extensos bancos ao longo de toda a costa brasileira, principalmente no litoral das regiões sudeste e sul. Esta alga possui vasta importância para a fauna e flora dos ambientes onde ocorre, além de possuir uma importante aplicabilidade econômica, principalmente como fonte de alginato. Portanto, estudos que conduzam ao desenvolvimento de planos viáveis de manejo são necessários. Este trabalho buscou quantificar bancos de Sargassum cymosum na Enseada de Armação do Itapocoroy, SC, e avaliar o rendimento da alga em alginato, considerando ramos reprodutivos e vegetativos separadamente. A composição da comunidade de algas associadas também foi analisada. A área total de cobertura dos bancos foi estimada em $14.137 \mathrm{~m}^{2}$, correspondendo a uma biomassa instantânea de 17,90 $( \pm 9,07)$ toneladas de Sargassum (massa úmida). A biomassa média de Sargassum variou de 142,64 $( \pm 34,25)$ a $214,76( \pm 131,68) \mathrm{g} \mathrm{ms} \mathrm{m}^{-2}$ (massa seca) entre os bancos. O rendimento em alginato obtido oscilou entre $18,63 \%$ e $25,00 \%$, sendo que os ramos reprodutivos da planta tendem a apresentar rendimentos mais elevados do que os vegetativos. As estruturas reprodutivas sozinhas renderam cerca de $30 \%$ do peso em alginato. Apesar de que investigações adicionais sejam imprescindíveis, estes resultados indicam um potencial para a explotação de Sargassum na costa de Santa Catarina.
\end{abstract}

Palavras-chave: Sargassum, biomassa, alginato, epífitas.

\section{Sargassum cymosum (PHAEOPHYCEAE) BEDS IN ARMAÇÃO DO ITAPOCOROY BAY, PENHA, SC: BIOMASS AND ALGINATE YIELD}

\begin{abstract}
The seaweed Sargassum occurs in large beds along Brazilian Coast, mainly in Southern and Southeastern areas. This seaweed is very important to local fauna and flora. It is also economically important mainly due alginate to production. Several kinds of approaches are needed to planning its sustainable management. This work aims to evaluate the size of subtidal Sargassum cymosum beds in Armação do Itapocoroy Bay (Penha, SC), quantifying its spatial variations on biomass and associated seaweed community, and also evaluate alginate yield separately for vegetative and reproductive branches, for each bed. The Sargassum beds area in Armação do Itapocoroy Bay reached $14,137 \mathrm{~m}^{2}$. Total estimated biomass in the bay was $17.90 \pm 9.07$ ton wet mass. Mean biomass ranged from $142,64( \pm 34,25)$ to $214,76( \pm 131,68) \mathrm{g}$ dry mass $\mathrm{m}^{-2}$ between the beds. The alginate yield ranged from 18.63 to $25.00 \%$, and reproductive branches yield was higher than vegetative branches yield. Reproductive structures alone yield about $30 \%$. Despite many additional works are needed, these results indicate potential for Sargassum exploitation in Santa Catarina Coast.
\end{abstract}

Key-words: Sargassum, biomass, alginate, epiphytes 


\section{INTRODUÇÃO}

As algas pardas do gênero Sargassum apresentam distribuição bastante ampla, principalmente nas regiões tropicais e subtropicais (Prince \& O'neal, 1979), com um número estimado de espécies entre 250 (De Wreede \& Jones, 1973) e 400 (Yoshida, 1983). Somente no litoral brasileiro, estima-se que haja cerca de onze espécies (Paula, 1988). São algas que dominam sobre outras espécies nos ambientes em que ocorrem, formando extensos e densos bancos, cobrindo desde regiões submareais com substrato areno-rochoso até o limite inferior das regiões de entre-marés de costões tanto abrigados como expostos (Critchley et al., 1991).

O gênero Sargassum é muito utilizado como fonte de alimento por diversos macroherbívoros (Eston \& Bussab, 1990). Meso-herbívoros, principalmente anfípodas e decápodas, são também muito abundantes nos bancos de Sargassum (Tararam et al., 1978). Os intrincados ramos e as folhas de Sargassum oferecem uma complexa estrutura para a fixação de ovos dos mais variados organismos, além de servir de proteção contra predadores a uma fauna associada diversa. Bancos expostos desta alga durante a maré baixa podem oferecer proteção a alguns animais contra a incidência direta de raios solares, não permitindo sua dessecação (Largo \& Ohno, 1993). Inúmeras espécies de algas epifíticas e epilíticas são dependentes diretas da presença de bancos de Sargassum (Paula \& Oliveira Filho, 1980).

Dentre as muitas formas de usos atribuídas pelo homem, a que desperta maior interesse comercial é a utilização de Sargassum como fonte de alginato. Este polissacarídeo está presente na parede celular das algas pardas e possui ampla utilização industrial (Lewis et al., 1988). Não há no Brasil produção de alginatos e o mercado é crescente. Só no ano de 1994 foram importadas em torno de 500 toneladas, que custaram cerca de 6,5 milhões de dólares (Oliveira, 1998).
A notável importância ecológica e econômica da alga parda Sargassum demanda a necessidade de estudos que ampliem o seu conhecimento. A presente investigação foi dirigida a diferentes bancos de Sargassum cymosum na Enseada de Armação do Itapocoroy, litoral Centro-Norte catarinense, região onde a alga demonstra ampla ocorrência. Buscou-se determinar a área de cobertu$\mathrm{ra}$, a biomassa total dos bancos, avaliar o rendimento em alginato e a composição da comunidade de algas associadas, procurando identificar possíveis variações espaciais dentro da enseada.

\section{MATERIAIS E MÉTODOS}

\section{Caracterização da área de estudo}

A Enseada de Armação do Itapocoroy (2644'S, 4839'W, Figura 1) situa-se no município de Penha e se constitui em uma área naturalmente protegida de ondas, ventos e correntes marítimas de maiores intensidades. 0 local possui profundidades inferiores a $15 \mathrm{~m}$ e a

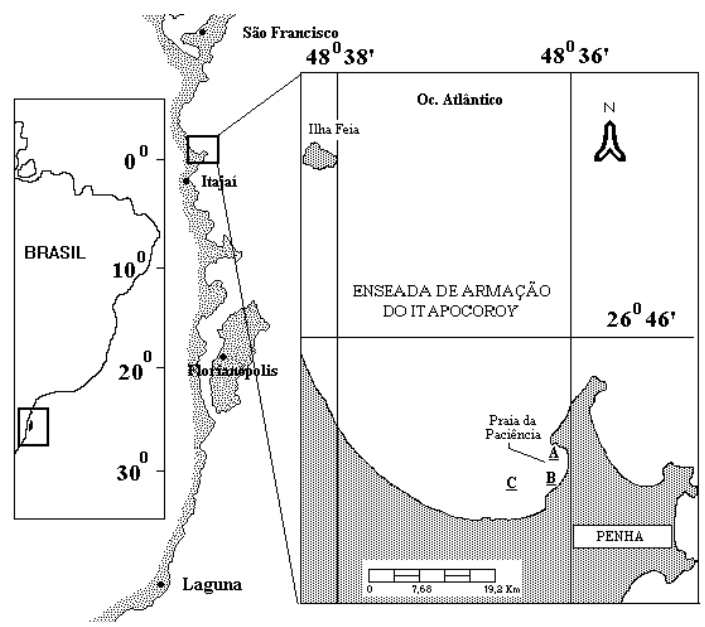

Figura 1 - Mapa da Enseada de Armação do Itapocoroy, SC, com destaque para a localização dos bancos "A", "B" e "C". 
média em torno de 8m. (Schettini et al., 1999). Os bancos de Sargassum estão distribuídos pela costa da enseada, em locais onde o substrato torna-se favorável à fixação da alga, com presença de fundo rochoso a areno-rochoso. O local abriga o maior parque nacional de cultivo de moluscos marinhos do Brasil.

\section{Biomassa total dos bancos}

Depois de realizados levantamentos preliminares, foram selecionados três bancos de Sargassum cymosum mais representativos em termos de área coberta, na Enseada de Armação do Itapocoroy. Estes foram denominados de Banco "A", Banco "B" e Banco "C", sendo os dois primeiros localizados na região rochosa da costa adjacente à Praia da Paciência, e o último em torno de uma saliência rochosa inserida na área destinada ao cultivo de moluscos na Praia de Armação do Itapocoroy (Figura 1).

Para se estimar a área coberta pelos mesmos, realizaram-se operações de merguIho autônomo com um grupo de quatro pessoas. Foram traçados perfis ortogonais dentro dos bancos, medindo-se com uma trena de $50 \mathrm{~m}$. A área total de cada banco foi calculada a partir de desenhos esquemáticos, provenientes das medidas obtidas em campo.

A poda de Sargassum cymosum para determinação da biomassa ocorreu entre $30 \mathrm{de}$ março e 05 de abril de 2001, que representa o final do verão e início do outono, período determinado em estudo prévio como o de biomassa mais elevada (Mafra Jr., 2001).

A coleta do material foi efetuada utilizando-se amostradores de $75 \mathrm{~cm} \times 75 \mathrm{~cm}$ (aproximadamente $0,58 \mathrm{~m}^{2}$ ), dentro dos quais todos os indivíduos foram cortados na base, próximo ao disco de fixação da alga, com auxílio de tesoura de jardinagem. Toda a comunidade de algas associadas ao banco de Sargassum cymosum também foi cortada na base. A amostragem contemplou sete pontos aleatórios em cada banco. O material coletado foi con- duzido ao laboratório para preservação por meio de congelamento e triagem.

Durante a triagem macroscópica em laboratório, a alga $S$. cymosum foi limpa e isolada de outros organismos e materiais associados. Depois de isolada, foi seca superficialmente com papel toalha e pesada em balança analítica para a determinação da massa úmida. Em seguida, o material foi o seco em estufa sob temperatura de $65^{\circ} \mathrm{C}$ até atingir um peso constante, que consistiu em sua massa seca. Os valores obtidos foram padronizados para as unidades de biomassa - gramas de massa úmida por metro quadrado $\left(\mathrm{g} \mathrm{mu} \mathrm{m}^{-2}\right)$ e gramas de massa seca por metro quadrado $\left(\mathrm{g} \mathrm{ms} \mathrm{m}^{-2}\right)$.

\section{Comunidade de algas associadas}

As algas associadas aos bancos de $S$. cymosum, depois de separadas na triagem, foram classificadas somente até o nível de gênero. Os gêneros de algas associadas foram pesados separadamente. Posteriormente, foi calculado um percentual de contribuição de cada gênero para a biomassa total da comunidade associada aos bancos de Sargassum cymosum.

\section{Extração de alginato}

A metodologia de extração empregada consistiu em uma adaptação da utilizada por Ragaza \& Hurtado (1999). Para a extração de alginato de cada amostra, foram adicionados 25 gramas de massa seca de Sargassum cymosum moído e $300 \mathrm{ml}$ de cloreto de cálcio (1\%). Depois de aquecida a mistura a $60^{\circ} \mathrm{C}$ durante 30 minutos, ela foi filtrada em rede de zooplâncton (malha de $300 \mu \mathrm{m}$ ), descartandose o líquido filtrado e repetindo-se o processo mais uma vez.

Então, ao resíduo sólido retido na rede adicionaram-se $300 \mathrm{ml}$ de ácido clorídrico (1\%). A mistura foi aquecida por 30 minutos a $60^{\circ} \mathrm{C}$ e, em seguida, filtrada. O procedimento foi repetido duas vezes, descartando-se o resíduo 
líquido. Logo após, foram adicionados $300 \mathrm{ml}$ de carbonato de sódio (1\%), ficando em repouso para digestão noturna.

No dia seguinte, a solução foi aquecida a $60^{\circ} \mathrm{C}$ durante duas horas e depois filtrada. $\mathrm{O}$ resíduo sólido foi descartado e o líquido filtrado foi branqueado com hipoclorito de sódio na proporção 2:1 (v/v) durante 30 minutos. O alginato foi precipitado com álcool etílico $(95 \%)$ na relação $1: 2(\mathrm{v} / \mathrm{v})$ e seco em estufa $\left(65^{\circ} \mathrm{C}\right)$ até atingir peso constante. Depois de seco, foi realizada a pesagem do alginato precipitado para calcular o seu rendimento, representado como percentual do peso original da alga.

O rendimento médio em alginato foi comparado entre os bancos, buscando-se determinar uma variação espacial. Os ramos reprodutivos (com estruturas reprodutivas - receptáculos) e vegetativos da alga foram separados para comparar os teores de alginato nessas porções distintas. Também foram selecionados somente os receptáculos de uma única amostra para uma avaliação experimental do teor de alginato nos mesmos.

\section{RESULTADOS}

\section{Biomassa total dos bancos}

Os bancos de Sargassum cymosum, denominados de "A", "B" e "C" (Figura 1), apresentaram características aproximadamente similares de densidade e tamanho individual das algas. O banco que apresentou a maior área de cobertura foi o "B", estimado em $7.067 \mathrm{~m}^{2}$, seguido do banco "A", com área de $3.650 \mathrm{~m}^{2} \mathrm{e}$ do banco "C", com $3.420 \mathrm{~m}^{2}$. No total, cobriram uma área de $14.137 \mathrm{~m}^{2}$.

O banco "B" teve uma biomassa média avaliada em 214,76 $( \pm 131,68) \mathrm{g} \mathrm{ms} \mathrm{m}^{-2}$, já a biomassa média do banco "A" foi estimada em $186,36( \pm 85,54) \mathrm{g} \mathrm{ms} \mathrm{m}^{-2} \mathrm{e}$ a do banco "C" em $142,64( \pm 34,25) \mathrm{g} \mathrm{ms} \mathrm{m}^{-2}$ (Figura 2A). A biomassa seca média ponderada para toda a enseada, considerando os três bancos, ficou em $189,98 \mathrm{~g} \mathrm{~ms} \mathrm{~m}^{-2}$ ou, em biomassa úmida, $1,27 \mathrm{~kg} \mathrm{mu} \mathrm{m}^{-2}$.

Extrapolando-se a biomassa média para a área total ocupada pelos bancos, tem-se a biomassa instantânea na área de estudo para o período de maior biomassa, o início do outono. O banco "B" foi o que teve a maior biomassa total estimada, reflexo da biomassa média mais elevada e da maior área de cobertura (Tabela 1). Os bancos somados resultaram em uma biomassa seca de 2,69 $( \pm 1,36)$ ton, ou em massa úmida, 17,90 $( \pm 9,07)$ ton.

\section{Comunidade de algas associadas}

A maior parte dos gêneros encontrados ocorreu como epífitas sobre Sargassum. Outros ocorreram sob a forma epilítica, ou seja, diretamente sobre o substrato. Foi o caso, por exemplo, do gênero Spatoglossum. O banco "A" foi o que demonstrou a maior presença de algas associadas, bem como a de incrustações de diversas naturezas, como algas vermelhas coralináceas não-articuladas, tubos de poliquetas, desovas não identificadas de invertebrados, entre outras. No banco "A", as algas associadas representaram, em média, $27,55 \%$ do peso de Sargassum cymosum e apresentaram uma biomassa média de 34,09 $( \pm 23,18) \mathrm{g} \mathrm{ms} \mathrm{m}^{-2}$. Já no banco "B", a proporção média destas algas em relação ao $S$. cymosum foi de $5,17 \%$ e o valor obtido de biomassa média foi de $8,32( \pm 9,61) \mathrm{g} \mathrm{ms} \mathrm{m}^{-2}$. No banco "C" as algas associadas representaram, em média, somente $1,75 \%$ do peso de $S$. cymosum. Este foi o banco que teve o valor mais baixo de biomassa média de algas associadas, reportada em 2,58 $( \pm 2,46) \mathrm{g} \mathrm{ms} \mathrm{m}^{-2}$ (Figura 2B).

Alguns gêneros principais contribuíram para o valor total de biomassa na comunidade associada aos bancos de Sargassum cymosum, tais como Bryothamnium, Dictyopteris, Galaxaura, Hypnea, Laurencia e Spatoglossum. Além destes, alguns gêneros apresentaram menores representatividades e 

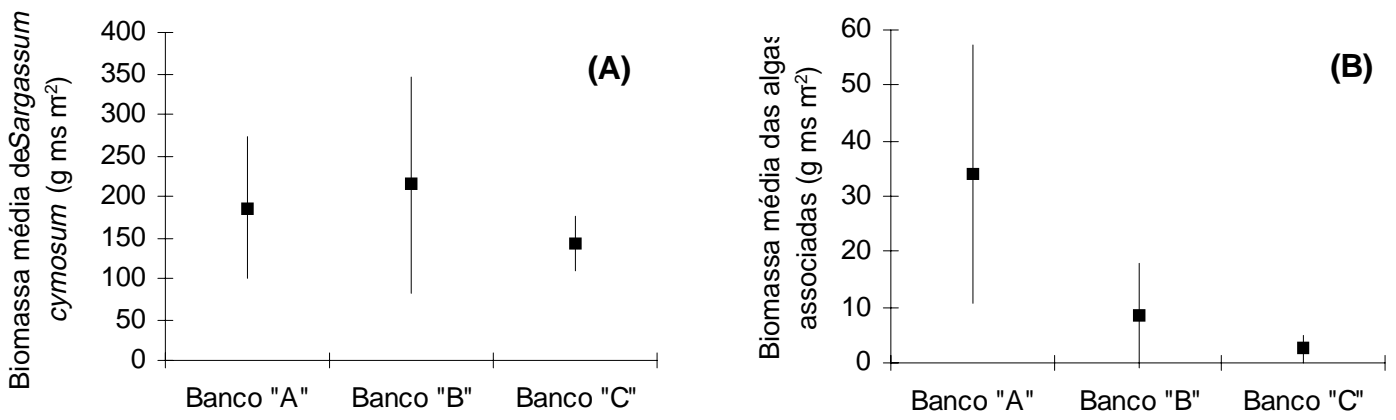

Figura 2 - Biomassa média de Sargassum cymosum (A) e das algas associadas (B) aos bancos na Enseada de Armação do Itapocoroy, SC, e desvio-padrão $\left(\mathrm{g} \mathrm{ms} \mathrm{m}^{-2}\right)$.

Tabela 1 - Biomassa média e total de Sargassum cymosum (massa seca e úmida), Enseada de Armação do Itapocoroy, SC.

\begin{tabular}{lrrrr}
\hline \multicolumn{1}{c}{ Área } & \multicolumn{2}{c}{ Massa seca } & \multicolumn{2}{c}{ Massa úmida } \\
& Médio $\left(\mathrm{g} \mathrm{m}^{-2}\right)$ & \multicolumn{1}{c}{ Total $(\mathrm{kg})$} & Médio $\left(\mathrm{g} \mathrm{m}^{-2}\right)$ & \multicolumn{1}{c}{ Total $(\mathrm{kg})$} \\
\hline Banco "A" & 186,36 & 680,22 & 1242,41 & 4534,81 \\
Banco "B" & 214,76 & 1517,68 & 1431,70 & 10117,84 \\
Banco "C" & 142,64 & 487,83 & 950,93 & 3252,18 \\
Todos & 189,98 & 2685,73 & 1266,52 & 17904,83 \\
\hline \hline
\end{tabular}

foram agrupados na categoria "outras algas", dentre eles Acanthophora, Bostrychia, Centroceras, Ceramium, Chondrachanthus, Dictyota, Gelidium, Gracilaria, Lobophora, Padina, Polysiphonia, Pterocladiella, Pterosiphonia e uma alga articulada da Família Coralinaceae. A biomassa total das algas associadas a cada banco investigado está apresentada na Tabela 2.

\section{Rendimento em alginato}

As extrações de alginato com Sargassum cymosum proveniente dos três bancos analisados resultaram em valores próximos de rendimento. As algas coletadas no banco "C" apresentaram maior rendimento $(23,36 \% \pm$
$4,08)$, em seguida as do banco "B" $(22,32 \% \pm$ $3,45)$ e do banco "A" $(21,24 \% \pm 2,58)$ (Figura 3). Considerando a biomassa total dos bancos, a quantidade de alginato virtualmente disponível para a explotação seria de 597,18 \pm 89,78 $\mathrm{kg}$ (massa seca).

Através das extrações isoladas em ramos reprodutivos, notou-se que os valores estimados de rendimento em alginato foram de $25,00 \%$ no banco "A", $24,84 \%$ no "C" e $23,80 \%$ no "B". Os ramos vegetativos, por sua vez, tiveram um rendimento estimado em $20,96 \%$ para o banco "C", 19,72\% para o "A" e 19,56\% para o "B" (Figura 3). Confrontando-se os resultados, percebe-se que os ramos reprodutivos apresentam um maior rendimento da biomassa em alginato do que os ramos vegetativos. Uma 
Tabela 2 - Contribuição percentual de cada gênero para a biomassa total de algas epífitas (kg ms) associadas a bancos de Sargassum cymosum na Enseada de Armação do Itapocoroy, SC.

\begin{tabular}{lcccc}
\multicolumn{1}{c}{$\begin{array}{c}\text { Algas } \\
\text { associadas }\end{array}$} & \multicolumn{2}{c}{ Peso total por banco (kg ms) } & Total \\
\hline Bryotamnium & $47,58(38,2 \%)$ & $20,89(35,5 \%)$ & & \\
Dictyopteris & $30,37(24,4 \%)$ & $23,74(40,4 \%)$ & $5,13(58,1 \%)$ & $58,47(35,7 \%)$ \\
Laurencia & $26,24(21,1 \%)$ & & & $26,24(13,7 \%)$ \\
Galaxaura & $15,72(12,6 \%)$ & & & $15,72(8,2 \%)$ \\
Spatoglossum & $1,50(1,2 \%)$ & $12,84(21,8 \%)$ & & $14,34(7,5 \%)$ \\
Hypnea & $2,38(1,9 \%)$ & $1,02(1,7 \%)$ & $1,94(21,9 \%)$ & $5,34(2,8 \%)$ \\
Outras algas & $0,65(0,5 \%)$ & $0,29(0,5 \%)$ & $1,76(20,0 \%)$ & $2,70(1,4 \%)$ \\
\hline Soma & $124,44(100 \%)$ & $58,78(100 \%)$ & $8,83(100 \%)$ & $192,05(100 \%)$
\end{tabular}

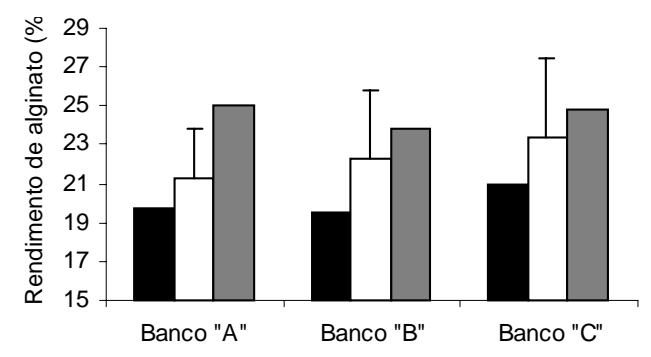

Ramos vegetativos $\square$ Alga inteira $\square$ Ramos reprodutivos

Figura 3 - Rendimento em alginato (\%) de Sargassum cymosum em três bancos da Enseada de Armação do Itapocoroy, SC, sob as formas de plantas inteiras, ramos reprodutivos e ramos vegetativos. Para a alga inteira, cuja extração foi feita com repetições, estão mostrados nos gráficos os desvios-padrão para o rendimento.

única extração de alginato utilizando somente os receptáculos apresentou um rendimento de $30,83 \%$.

\section{DISCUSSÃO}

Os três bancos avaliados na região da Enseada de Armação do Itapocoroy apresentaram diferenças entre os valores de suas biomassas médias, ou seja, a biomassa de alga por área de substrato. A variação entre os substratos sobre o qual estão assentados os bancos estudados pode ser um dos fatores responsáveis pelas diferenças na biomassa média, já que a alga parda Sargassum cymosum mostrou depender de um substrato rochoso para se fixar, por meio de seus sistemas apressórios. Desta forma, a disposição e a proporção de afloramentos rochosos no substrato, bem como de blocos soltos, são fatores determinantes na densidade dos indivíduos no banco.

Outros fatores responsáveis pela variação espacial na biomassa média dos bancos podem ser as características físicas locais, principalmente energia de onda e circulação local, embora aspectos como luminosidade, disponibilidade de nutrientes e interações biológicas possam representar fatores importantes para a densidade e biomassa dos bancos. Na costa sudeste brasileira, Széchy (1996) afirmou que locais moderadamente expostos e moderadamente protegidos da ação de ondas e correntes parecem ser mais favoráveis ao desenvolvimento de Sargassum. É nestes ambientes que a alga revela seus valores máximos de biomassa. 
Considerando os três bancos de Sargassum analisados, os valores de biomassa média ponderada e biomassa total para a enseada podem ser comparados a estudos realizados por autores distintos em algumas regiões da costa mexicana (Tabela 3). Tendo em vista que foram avaliados na presente investigação somente três bancos, em uma área geográfica relativamente pequena, os valores obtidos permitem supor que esta alga pode ser vista como um recurso potencial, especialmente considerando que o gênero Sargassum é bastante abundante ao longo do litoral de Santa Catarina (Ouriques, 1997). Sendo assim, espera-se que a partir do momento que outras áreas sejam mapeadas e quantificadas, o potencial real de Sargassum como recurso possa ser mensurado.

Com relação ao teor de alginato, embora tenham sido observadas pequenas variações espaciais entre os bancos da Enseada de Armação do Itapocoroy (Penha, SC), estas variações não são significativas. Em geral, o rendimento do gênero Sargassum em alginato apresenta-se bastante variável na literatura, dependendo da espécie abordada, metodologia aplicada, estágio reprodutivo, área geográfica, e/ ou condições ambientais.

Os maiores rendimentos de ramos reprodutivos em alginato em comparação aos ramos vegetativos observados no presente trabalho foram também observados por Ragaza \& Hurtado (1999), que analisou plantas reprodutivas e vegetativas isoladamente, e não em porções distintas de uma mesma planta.Isso certamente ocorre devido ao alto teor de alginato das estruturas reprodutivas, estimado em $30,83 \%$, em uma extração experimental no presente estudo.

Os valores de rendimento de Sargassum cymosum em alginato observados neste trabaIho (18 a $25 \%$ ) ficaram próximos aos citados na literatura para o gênero, tais como o rendimento encontrado por Chennubhotla et al. (1982), que oscilou entre 22,3 e $30,8 \%$ em Sargassum myriocystum. Guevara e Palma (1983) obtiveram variações no conteúdo de alginato entre $18,7 \%$ e $20,4 \%$ e Omar et al. (1988) encontraram valores entre 32 e $35 \%$. Ragaza \& Hurtado (1999) testaram o rendimento do alginato de três espécies de Sargassum, obtendo valores entre 10 e $41 \%$. Apesar dos rendimentos terem sido satisfatórios, faz-se necessária uma análise da qualidade do alginato proveniente de Sargassum cymosum na Enseada de Armação do Itapocoroy, SC.

Os resultados produzidos neste estudo evidenciam o potencial que Sargassum apresenta como recurso a ser explotado. Entretanto muitos outros fatores devem ser avaliados antes de se formalizar uma proposta de manejo. Além de determinar as áreas de maior biomassa e maior potencial produtivo, um bom plano de manejo deve considerar a intensidade, o período e a freqüência das podas ou coIheitas, a variação temporal da biomassa, a

Tabela 3 - Dados comparativos da biomassa média e total de Sargassum em diferentes estudos, confrontando com o atual, na Enseada de Armação do Itapocoroy, Penha, S.C.

\begin{tabular}{lccl}
\hline \hline \multicolumn{1}{c}{ Autor } & $\begin{array}{c}\text { Biomassa média } \\
\left(\mathrm{kg} \mathrm{mu} \mathrm{m}^{-2}\right)\end{array}$ & $\begin{array}{c}\text { Biomassa total } \\
(\text { ton. massa úmida) }\end{array}$ & \multicolumn{1}{c}{ Local } \\
\hline $\begin{array}{l}\text { Presente estudo } \\
\text { Hernández-Carmona et al. }\end{array}$ & 1,27 & 17,91 & Ens. Armação do Itapocoroy \\
$(1990)$ & & 18,90 & Bahía de La Paz, México \\
Casas-Valdez et al. (1993) & 3,42 & 7,25 & Bahía Concepción, México \\
Pacheco-Ruíz et al. (1998) & 5,00 & $8.256,00$ & Baja California Sur, México \\
Pacheco-Ruíz et al. (1998) & 16,40 & $146.303,00$ & Baja California, México \\
\hline \hline
\end{tabular}


sazonalidade do ciclo reprodutivo, a variação temporal do conteúdo de polissacarídeos e a técnica de colheita (Santelices, 1989). Vários destes aspectos foram abordados para $S$. cymosum na Enseada de Armação do Itapocoroy por Mafra Jr. (2001). Todavia, no caso das algas pardas, que formam bancos com grande abundância e diversidade de fauna e flora associadas, são necessários estudos que incluam os efeitos da poda sobre estas comunidades, tais como: densidade populacional; morfologia das plantas; competição na flora associada e processos de sucessão pós-poda; abundância da fauna associada e; vulnerabilidade dos bancos a distúrbios físicos naturais (Vasquez, 1995). Portanto, um plano de manejo dirigido a um recurso natural como a alga Sargassum deve abranger todos estes fatores, para que ele possa predizer os prejuízos ambientais e os benefícios sócio-econômicos acarretados pela atividade de explotação, garantindo sua sustentabilidade.

\section{REFERÊNCIAS BIBLIOGRÁFICAS}

Casas-Valdez, M.M.; Rodríguez, I.S. \& G. Hernández-Carmona. 1993. Evaluación de Sargassum spp. en la Costa Oeste de Bahía Concepción, B.C.S., México. Inv. Mar. CICIMAR. 8: 61-69.

Critchley, A.T.; Peddemors, V.M. \& R. Pienaar. 1991. Reproduction and establishment of Sargassum heterophyllum (Turner) C. Ag. (Phaeophyceae, Fucales). Br. Phycol. J., Glasgow. 26: 303-314.

Chennubhotla, V.S.K.; Kaliaperumal, N.; Kalimuthu, S.L; Selvaraj, M; Ramaligaon, J.R. \& M. Najmuddin. 1982. Seasonal changes in growth and alginic acid and mannitol contents in Sargassum ilicifolium and S. myriocy. Indian-J. Mar. Sci. 11: 195196.

De Wreede, R.E. \& E.C. Jones. 1973. New records of Sargassum hawaiensis (Sargassacea, Phaeophyta), a deep water species. Phycologia la. 1 (12): 59-62.
Eston, V.R. \& W.O. Bussab. 1990. An experimental analysis of ecological dominance in a rocky subtidal macroalgal community. J. exp. mar. Biol. Ecol., Amsterdam. 136: 170-195.

Guevara, G. \& H. Palma. 1983. Alginate contents in two species of brown algae, Padina gymnospora and Sargassum filipendula, from the coasts of Margarita Island, Venezuela. Cont. Estac. Invest. Mar. Margarita. 110: 9-27.

Hernandez-Carmona, G.; Casas Valdez, M.; Leon, C.F.; Sanchez-Rodriguez, I. \& E. Rodriguez-Montesinos. 1990. Evaluación de Sargassum spp. en la Bahía de La Paz, B.C.S., México. Inv. Mar. CICIMAR. 5: 1118.

Largo, D.B. \& M. Ohno. 1993. Constructing an artificial seaweed bed, p. 113-130. In: M. Ohno \& A.T. Critchley [Eds.], Seaweed cultivation and marine ranching. Japan International Cooperation Agency (JICA).

Lewis, J.G.; Stanley, N.F. \& G. G. Guist. 1988. Commercial production and applications of algal hydrocolloids, p. 205-237. In: C.A. Lembi \& J.R. Waaland [Eds], Algae and Human Affairs. Cambridge.

Mafra Jr., L.L. 2001. Bases para o manejo de Sargassum cymosum (Phaeophyta Fucales) na Enseada de Armação do Itapocoroy, Penha, SC. Monografia de Graduação, Universidade do Vale do Itajaí. 61 pp.

Oliveira, E.C. 1998. The seaweed resources of Brazil, p. 366-371. In: M. Ohno \& A.T. Critchley [Eds.], Seaweed cultivation and marine ranching. Japan International Cooperation Agency (JICA).

Omar, S.; Ahmad, N. \& F. Ahmad. 1988. Composition of alginates from brown seaweeds, Sargassum and Padina spp. Pertanika. 11: 79-85.

Ouriques, L.C. 1997. Feofíceas do litoral do Estado de Santa Catarina. Dissertação de Mestrado, Universidade Estadual Paulista. 254 pp. 
Pacheco-Ruíz, I.; Zertuche-Gonzáles, J.A.; Chee-Barragán, A. \& R. BlancoBetancourt. 1998. Distribution and quantification of Sargassum beds along the West Coast of the Gulf of California, Mexico. Bot. Mar. 41: 203-208.

Paula, E.J. 1988. O gênero Sargassum (Phaeophyta - Fucales) no litoral do Estado de São Paulo, Brasil. Bolm. Botânica, Univ. S. Paulo. 10; 65-118.

Paula, E.J. \& E.C. Oliveira Filho. 1980. Aspectos fenológicos de duas populações de Sargassum cymosum (Phaeophyta Fucales) do litoral de São Paulo, Brasil. Bolm. Botânica, Univ. S. Paulo. 8: 21-39.

Prince, J.S. \& S.W. O'neal. 1979. The ecology of Sargassum pteropleuron in the waters off South Florida. I. Growth, reproduction ands population structure. Phycologia. 18: 109-114.

Ragaza, A.R. \& A.Q. Hurtado. 1999. Sargassum studies in Currimao, llocos Norte, Northern Philippines. II. Seasonal variations in alginate yield and viscosity of Sargassum carpophillum J. Agardh, S. ilicifolium (Turner) C. Agardh and $S$. siliquosum J. Agardh (Phaeophyta, Sargassaceae). Bot. Mar. 42: 327-331.

Santelices, B. 1989. Algas marinas de Chile. Distribuición, Ecologia, Utilización, Diversidad. Ed. Universidad Católica de Chile. Chile. 399 pp.

Schettini, C.A.F.; de Carvalho, J.L.B \& E. C. Truccolo. 1999. Aspectos hidrodinâmicos da Enseada da Armação do Itapocoroy, SC. Notas Técnicas da Facimar. 3: 99-109.

Széchy, M.T.M. 1996. Estrutura de bancos de Sargassum (Phaeophyta-Fucales) do litoral dos Estados do Rio de Janeiro e São Paulo. Tese de Doutorado, Universidade de São Paulo. 355 pp.

Tararam, A.S.; Wanabara, Y. \& A.M. Takeda. 1978. Seasonal variations of amphipoda species living on Sargassum in Itanhaem, São Paulo - Brazil. Contribuition no 501 of Inst. Ocean., Univ. de São Paulo.

Vasquez, J.A. 1995. Ecological Effects of Brown Seaweed Harvesting. Bot. Mar. 38: 251-257. Yoshida, T. 1983. Japanese species of Sargassum subgenus Bactrophycus (Phaeophyta - Fucales). J. Fac .Sci Hokkaido Univer,. ser. V (Botany). 13: 99246. 